\title{
Effective macroion charge and stability of highly asymmetric electrolytes at various salt conditions
}

\author{
Vladimir Lobaskin* \\ Physics Department, University of Fribourg, CH-1700 Fribourg, Switzerland \\ Khawla Qamhieh \\ Physics Department, College of Science and Technology, Al-Quds University, Jerusalem, Palestine
}

(Dated: May 6, 2019)

\begin{abstract}
We study electrostatic mechanisms of destabilization of highly asymmetric electrolytes. For this purpose, we perform primitive model Monte Carlo simulations of charged macroions immersed in multivalent salt solution. At low salt concentration, the macroion effective charge is reduced due to multivalent counterion adsorption. At high salt concentrations, the macroions become overcharged so that their apparent charge has the opposite sign to the stoichiometric one. The inverted charge is growing up to a saturation value upon further increasing the salinity. The system remains stable at low as well as at very high salt concentrations. In the intermediate region, close to the macroion isoelectric point, we observe macroion aggregation. The obtained phase behaviour closely resembles polyelectrolyte-induced instability of colloidal dispersions.
\end{abstract}

\section{INTRODUCTION}

Electrostatic interactions in colloidal suspensions and their relation to their phase stability have always been attracting much attention because of their occurence in many biological and technological circumstances. It has been known for a long time that presence of the oppositely charged polyions seriously deteriorates the stability of colloidal dispersions. In polyelectrolyte-induced aggregation, a correspondence between the location of the fast aggregation regime and the colloid isoelectric point has been documented repeatedly [1, 2, 3, 4]. Furthermore, the electrophoretic mobility of colloidal particles, which reflects the apparent particle charge, has been shown to be tightly correlated with the system's stability. The attractive interactions due to surface charge heterogeneities have been thought to promote colloidal aggregation in colloid-polyelectrolyte systems ("charge patch flocculation") 1, 2, 4]. It however only recently became clear that apart from specific binding between different solutes and van der Waals forces, the electrostatic correlations themselves can lead to variety of exciting phenomena in systems contaning oppositely charged polyions such as giant charge inversion or short-range attraction between like-charged polyions (meaning either colloids or polyelectrolytes) [5, 6, 7, 8, 9, 10, 11, 12, 13, 14, 15, 16, 17, 18, 19, 20. Moreover, computer simulations and theoretical studies have revealed that the correlation induced attraction can cause colloidal aggregation even when just small multivalent ions are present $8,12,13,16,21$. It was shown that the universality of the underlying phenomena is based on the enormous energetic contribution of the Coulombic interactions to the solution's free energy, which dominates over the entropy and makes the system unsensitive to the internal structure of the counterions [10, 13, 22, 23, 24, 25]. A phase diagram featuring a phase separation region around the macroion isoelectric point with a consequent re-stabilization was predicted for a purely Coulombic system containing polyions and a sufficient amount of multivalent counterions [15, 26].

In the present work, we present a computer simulation study of charge inversion and phase instabilities in an asymmetric electrolyte treated by a multivalent salt. This model is meant as a generic representation of a solution containing two types of oppositely charged polyions. We hope to recover the basic properties of such a system originating from the electrostatics and translational degrees of freedom of the solutes. Our simulation setup resembles a common experimental situation when an initially stable suspension is destabilized by adding oppositely charged multivalent ions (or polyelectrolyte) and aimed to outline the range of activity of the "patch charge attraction" [1, 2, 4] as well as assess the theoretical predictions [15, 26, 27] for phase behaviour of asymmetric Coulombic systems at different electrostatic coupling parameters. This work also complements the recent computer simulation studies of these phenomena [21, 24, 25, 28] by the investigation into salt effects and the role of the effective macroion charge.

The paper is organized as follows. In Section IIA we give the description of the model and parameter settings for the numerical experiment. Section ЏII gives a detailed account of the results for three series of simulations with different charge asymmetries. Section IV presents discussion of the mechanisms of observed phase behaviour and its relation to effective interactions between the colloids. The conclusions are given in the final section.

\footnotetext{
* Present address: Max Planck Institute for Polymer Research, D-55128 Mainz, Germany. e-mail: Lobaskin@mpip-mainz.mpg.de
} 


\section{MODEL AND METHOD}

\section{A. Model}

The systems under consideration are asymmetric electrolytes described within the framework of the primitive model. The reference electrolyte contains two types of spherical charged particles: (i) macroions of diameter $\sigma_{M}=40 \AA$ and charge $Z_{M}=-60$ and (ii) small ions of diameter $\sigma_{I}=4 \AA$ and charge $Z_{I}=+1$, representing the counterions, whereas the solvent enters the model only by its relative dielectric permittivity $\varepsilon_{r}$. The added salt consists of small ions of diameter $4 \AA$ : monovalent anions $Z_{a}=-1$ and cations of different valencies. We classified the simulated systems by the added cation charge: the series $\mathrm{A}$ contains cations with $Z_{c}=+1$, the series $\mathrm{B} Z_{c}=+3$, and the series $\mathrm{C} Z_{c}=+5$. The two last chosen coupling strengths belong to the instability region on the generic phase diagram for the system including only macroions with their counterions [12, 28]. The initial 60:1 system was previously studied in [28, 29] and in deionized system with trivalent counterions a phase separation was found 12, 21]. We characterize the amount of added salt by a ratio of the overall added cation charge to the overall macroion charge, $\beta=Z_{c} \rho_{c} /\left(Z_{M} \rho_{M}\right)$, where $\rho_{i}$ is the number density of the corresponding species.

The interaction between the particles in our model is pairwise additive, and for pair $i j$, where $i$ and $j$ denote either polyion or counterion, it is given by

$$
U_{i j}(r)=\left\{\begin{array}{cc}
\infty, & r<\left(\sigma_{i}+\sigma_{j}\right) / 2 \\
\frac{Z_{i} Z_{j} e^{2}}{4 \pi \epsilon_{0} \epsilon_{r}} \frac{1}{r}, & r \geq\left(\sigma_{i}+\sigma_{j}\right) / 2
\end{array}\right.
$$

where $r$ is the center-to-center separation between the particles.

To characterize the intensity of the electrostatic correlations between counterions on the surface, we use the counterion-counterion coupling parameter $\Gamma=Z_{I}^{2} l_{b} / a_{Z}$, where $l_{b}=\left(e^{2} / 4 \pi \varepsilon_{0} \varepsilon_{r} k T\right)$ is the Bjerrum length, $e$ is the elementary charge, $\varepsilon_{0}$ the dielectric permittivity of vacuum, and $a_{Z}=\left[Z_{I} /(\sigma / e)\right]^{1 / 2}$ the average distance between two neighboring counterions at the charged surface characterized by the surface charge density $\sigma$. It is known that the correlation-induced attraction appears at $\Gamma>\Gamma^{*} \approx 2[7,21,28]$. Our systems $\mathrm{A}, \mathrm{B}$, and $\mathrm{C}$ correspond to $\Gamma=0.8$, 4.1 , and 8.8, respectively.

The systems are considered at a fixed macroion number density $\rho_{M}=2.5 \cdot 10^{-7} \AA^{-3}$ corresponding to a macroion volume fraction $\phi_{M}=0.0084$. A temperature of $T=298 \mathrm{~K}$ and a relative dielectric permittivity of $\varepsilon_{r}=78.4$ were used. For each of the three series, 11 values of $\beta$ ranging from 0.1 to 10 were studied.

\section{B. Method and simulation settings}

In this study, we applied multiparticle Monte Carlo (MC) simulation method, which was developed in our earlier works [29, 30]. The canonical NVT ensemble with periodic boundary conditions and the Ewald summation for handling the electrostatic interactions were employed. The simulated bulk systems were composed of 20 macroions, 1200 monovalent counterions, and various amounts of added salt ions. Typically, $0.5-1 \cdot 10^{5}$ attempted MC moves per particles were made in the production runs, while for samples at $\beta \approx 1$ we performed up to $1 \cdot 10^{7}$ moves.

For the simulation of solutions with much salt we combined cluster MC moves for macroions [29] with swap moves, which is a modified implementation of the cluster moves. In the swap move, we first select the macroion and determine the particles within a concentric sphere around it. Then a second sphere of the same radius is taken centered at the destination vector of the macroion translational move $\delta \mathbf{r}_{M}$ and a central inversion of the content of the whole volume within the two (possibly overlapping) spheres about the center of symmetry at $\delta \mathbf{r}_{M} / 2$ is performed. The acceptance ratio is calculated then in the same way as for the standard cluster move [29], which results for a hard-sphere system and with $100 \%$ probability of the particle inclusion in the cluster in rejection of the moves that change the number of particles in the cluster (cf. discussion in 29]). This type of move significantly improves the acceptance ratio for the systems with homogeneously distributed ions in the bulk as the macroions always move into an ion-free volume.

To evaluate the effective macroion charge and the osmotic coefficient, we applied the spherical Wigner-Seitz (WS) cell model. The model was solved by canonical MC simulations for one centrally placed macroion with corresponding amount of small ions, while the other settings were the same as for the bulk simulation. The simulation package MOLSIM v3.1 by Per Linse et al. was used throughout. Further details of the simulation protocol can be found in Refs. [29, 30]. 


\section{RESULTS}

\section{A. Single-macroion properties. Thermodynamics}

We start discussing the numerical results from thermodynamic properties of the chosen electrolytes as function of the salt concentration. In Figs. 12 we plotted the reduced electrostatic energy of the solution $U / N k_{B} T$, and the osmotic coefficient $\Pi / \rho k_{B} T$, where $N$ and $\rho$ are the total number and the number density of the ionic species, respectively, $k_{B}$ the Boltzmann constant, $\Pi$ the osmotic pressure. The reduced energy was calculated both from bulk simulation and WS cell model. The Fig. 11shows that the total potential energy is negative at all the studied conditions as a result of strong attraction between the macroions and counterions. Its magnitude is decreasing monotonously with the salt content, which is a simple consequence of increasing the overall number of particles. All the curves have similar shape with varying curvature. An inflection point is observed at about $\beta=1$ on each curve. For the osmotic coefficient shown in Fig. 2] we present the cell model results obtained from the total density of ions at the cell boundary $\Pi_{\text {cell }} /\left(\rho k_{B} T\right)=\rho\left(R_{\text {cell }}\right) / \rho$. The osmotic coefficient behaves differently for systems A, and B and C. For the system $\mathrm{A}$, it is increasing roughly proportionally to $\ln \beta$ up to $\beta=1$, and after that is more slowly approaching unity, the ideal solution value. In the two latter series it is again growing proportional to $\ln \beta$ at low salt but with slightly higher coefficient. Both curves for series $\mathrm{B}$ and $\mathrm{C}$ have a peak at $\beta=1$ and then decrease proportionally to $-\ln \beta$, the decay of series $\mathrm{C}$ results being faster. The value of the osmotic coefficient at the peak is about 0.8. The proximity of the $\beta$ values to unity in all these cases reflects the high fraction of the free ions in the solution. This fraction can be roughly estimated as $\left(N_{a}+N_{c}+Z_{e f f}+N_{M}\right) / N$, which gives at $\beta=1$ for complete adsorption of the added cations $121 / 181,121 / 141$, and $121 / 133$, or $0.67,0.86$, and 0.91 for the systems A, B, and C, respectively. The further decrease of the osmotic coefficient is a result of association of salt anions with the multivalent cations, which reduces the number of "free" species as compared to the ideal solution value.

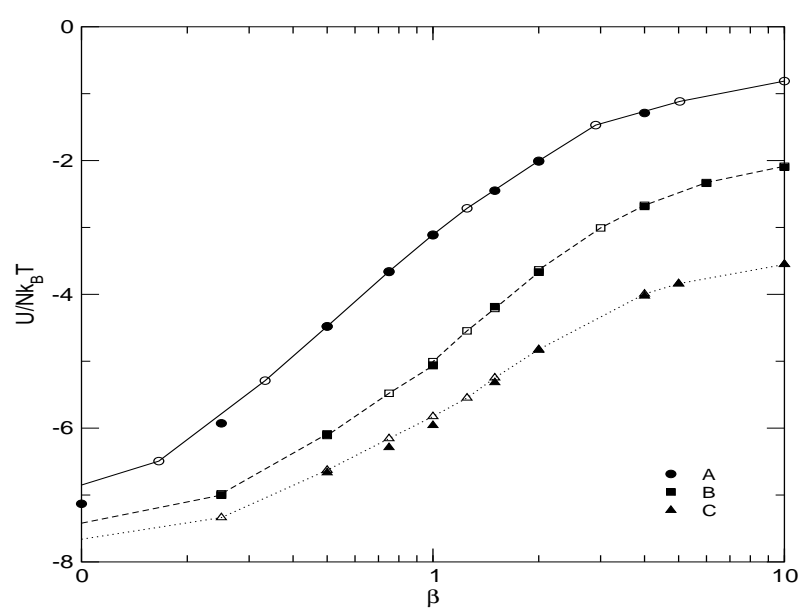

FIG. 1: Reduced electrostatic energy for series A, B, and C from bulk MC simulation with 20 macroions (full symbols) and the corresponding WS cell model (open symbols).

\section{B. Single-macroion properties: Effective charge}

The concept of effective polyion charge attracted much attention in literature due to its importance for the reduction problem. It immediately arises whenever the solvent- or ion-averaged interactions between solutes are concerned. The two major trends (excluding the experimental definitions) for two-component systems (macroion and counterions) are represented by the thermal and structural definitions. The different definitions in a spherically symmetric case regard the distance from the macroion center, within which the charge should be counted. The structural definitions are based on the knowledge of the radius, at which the mixed macroion-ion rdf falls below unity, or other criteria. The simplest of the thermal definitions counts the charge inside the macroion-ion binding radius $\left(\left|U\left(r_{\text {therm }}\right)\right|=k T\right)$. The more accurate one considers the chemical potential of the counterion in the macroion field. In the cylindrical case it was developed by Manning and Oosawa [31, 32] and adopted for spherical geometry by Belloni [33]. The radius in this 


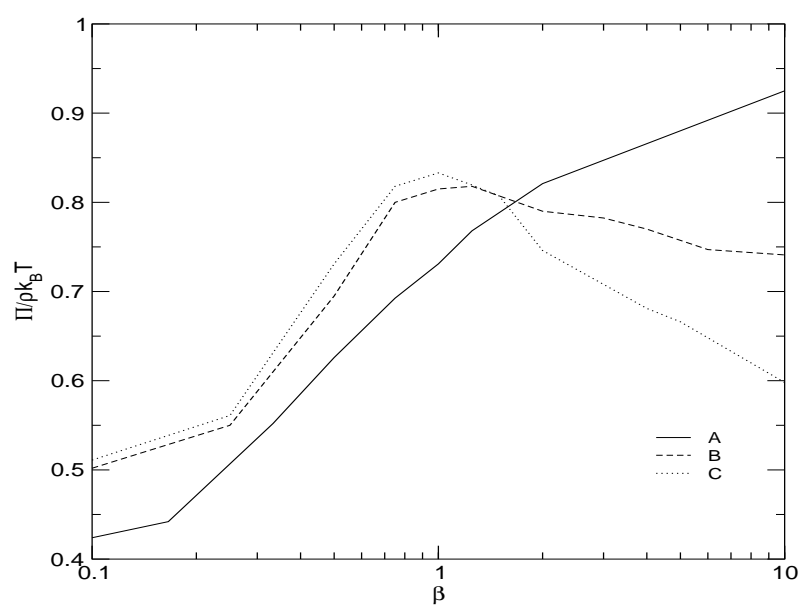

FIG. 2: Osmotic coefficient $\left(\rho\left(R_{\text {cell }} / \rho\right)\right)$ for series A, B, and C from MC simulation of the WS cell model.

approach is measured at the inflection point of the accumulated running charge curve plotted over inverse distance from the macroion center $1 / r$ (see also refs. [33, 35] for discussion of alternative definitions). Obviously, the radius is different for the ions of different valencies.

In the case of multicomponent electrolyte, however, we have to apply different definitions to different ionic species due to qualitatively dissimilar behaviour of the integrated running charge depending on the salt valency. Here, we use MC simulation to solve the WS cell model 29] and then extract the effective charge out of the integrated running charge curves. For series A and series B, C at low salt, we apply the inflection point method [33]. For the At $\beta>1$, where the inflection point was not accessible anymore, we evaluated the effective charge at the first extremum of the integrated running charge curves.

The Fig. 3 demonstrates typical ionic distribution profiles for systems A, B and C. While all the curves decay monotonously in the series $\mathrm{A}$, the systems $\mathrm{B}$ and $\mathrm{C}$ display diverse behaviour for the different ionic species. The number density of monovalent counterions decays similarly to the system A. The multivalent ion density has a high maximum at the polyion surface, while farther in the bulk it is close to zero. The coion distribution is peaked close to the maximum of the multivalent ion distribution because of their attraction to the macroions with the inverted charge and becomes very pronounced at the high salt concentrations $\underline{36}$.

The integrated running charge curves are presented in Fig. 4 There is a distinct qualitative difference between system A and two other systems B, C. While in the former one the total charge is decaying monotonously and reaches zero only at the cell boundary, in the two latter systems the charge exhibit a sharp drop to zero within a very narrow region $r<1.2 \sigma_{M}$. At $\beta=1$, the running charge remains close to zero up to the cell boundary. At the higher $\beta$, the charge is changing its sign and after that decaying slowly. Close to the cell boundary, one can see the cation charge build-up (note that the charge is divided by the negative $Z_{M}$ ), which originates from repulsion between the charge inverted macroions and cations. Note that the accumulated charge in series A at high salt concentrations reaches zero already at $r=3 \sigma_{M}$, which is much less than the mean distance between the macroions.

In Fig. 5. we present the effective macroion charge as a function of $\beta$ for all three series. For all the systems $Z_{M}^{\text {eff }} / Z_{M}$ starts from about 0.3 at low added salt (its value for the 60:1 electrolyte [29]) and then monotonously decreases. The effective charge changes its sign for systems $\mathrm{B}$ and $\mathrm{C}$ and regains roughly the same magnitude $(0.17$ for series B and 0.36 for series $\mathrm{C}$ ) on the negative side. It is interesting to note the qualitative similarity of all the three curves. All of them have two relatively flat regions at very low and very high salt concentrations and a region of fast variation with an inflexion point at about $\beta=1$. The curve for series $\mathrm{A}$ asymptotically approaches but never crosses the zero line. Very slow dependence of the effective charge on the salt content at $\beta>1$ follows from logarithmical increase of the counterion entropy with counterion number density [26]. One should note that the decrease of the effective charge with the salt concentration, as it is seen for $\beta<1$, is characteristic for dilute and deionized systems, where the effective charge is far from its saturated value [33]. At the higher monovalent salt content, $\beta>1$, the ionic double layer saturates and $Z_{M}^{\text {eff }} / Z_{M}$ starts growing with salt concentration towards unity [33, 34]. We could not see this upturn due to the failure of the inflection point criterion in this region. The failure is due to the fact that the underlying division of the ionic population into the "condensed" and "free" parts is no longer valid. The inflection 

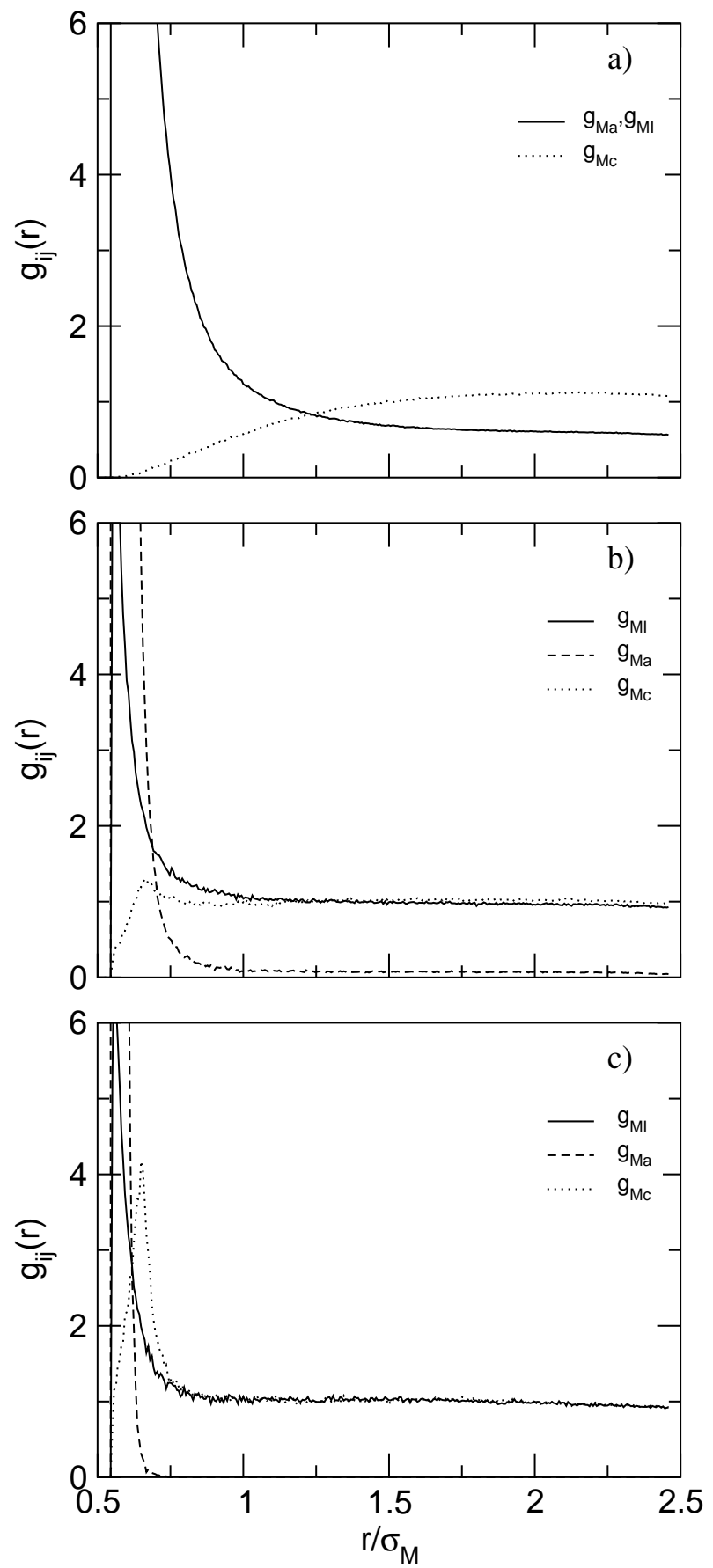

FIG. 3: Counterion $\left(g_{M I}\right)$ and added salt ion distribution functions (multivalent counterion $g_{M c}$, coion $g_{M a}$ ) for the series A, $\mathrm{B}$, and $\mathrm{C}$, respectively, as obtained from MC solution of the WS cell model.

point can still be detected formally but it refers now to the far field properties of the double layer (the inflection point is located next to the cell boundary) and does not reflect its short-range decay, which is important for the interaction between the macroions. 

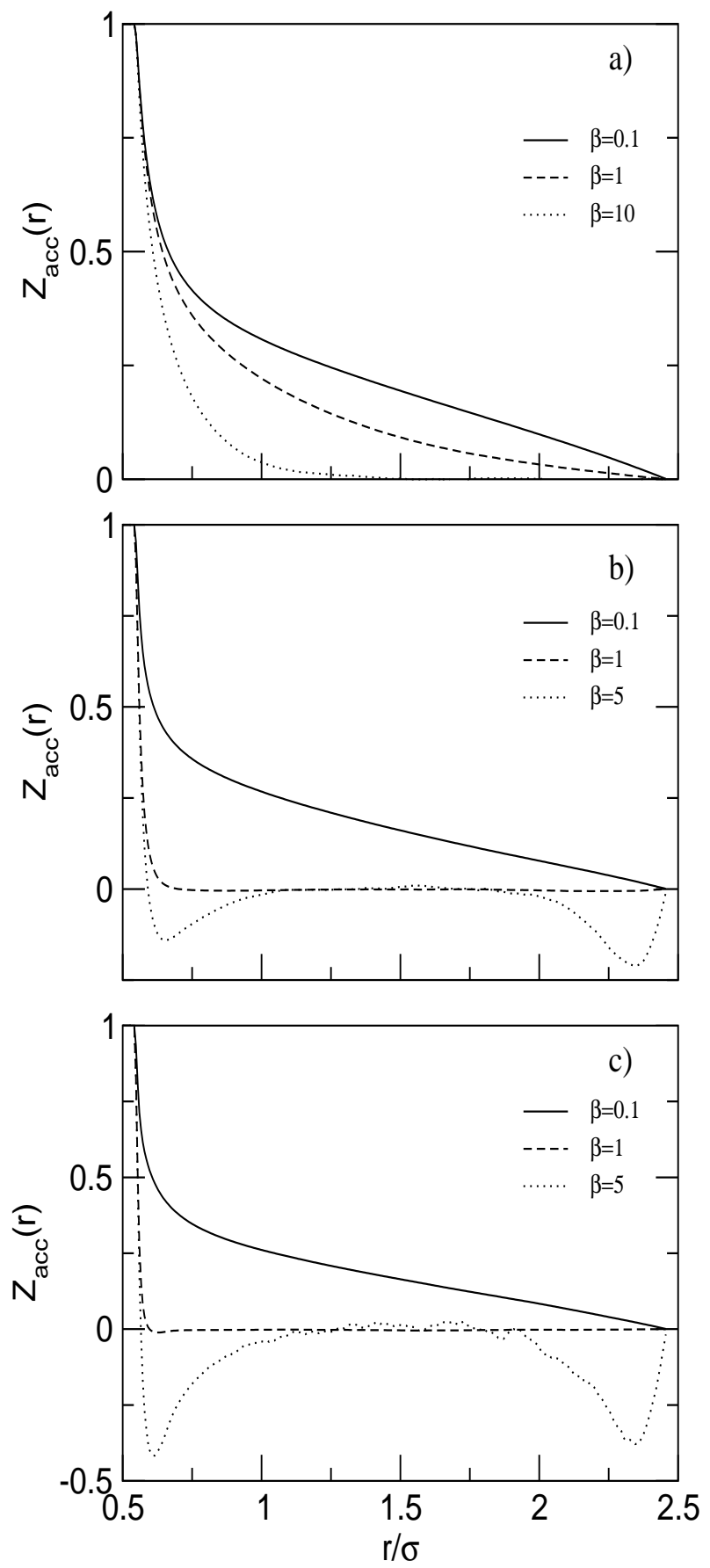

FIG. 4: Accumulated running charge as a function of the distance from the macroion center as obtained from MC solution of the WS cell model, a) series A, B series B, c) series C.

\section{Collective macroion properties: Structure}

The object of our main interest here is the macroionic distribution. We therefore analyse only macroion-macroion partial distributions and extract those data that characterize the macroion density fluctuations. Namely, we consider the long-wave limit value of the macroion-macroion partial structure factor $S_{M M}(q)$, which would quantify the reduced osmotic compressibility in a corresponding one-component fluid of macroions. We should note that due to the small size of the simulated sample we can access only relatively short-wave region $\left(q \sigma_{M}>0.01\right)$ and therefore have only rough estimate of $S_{M M}(0)$. One should expect stronger deviations from unity for the true $S_{M M}(0)$, i.e. even lower 


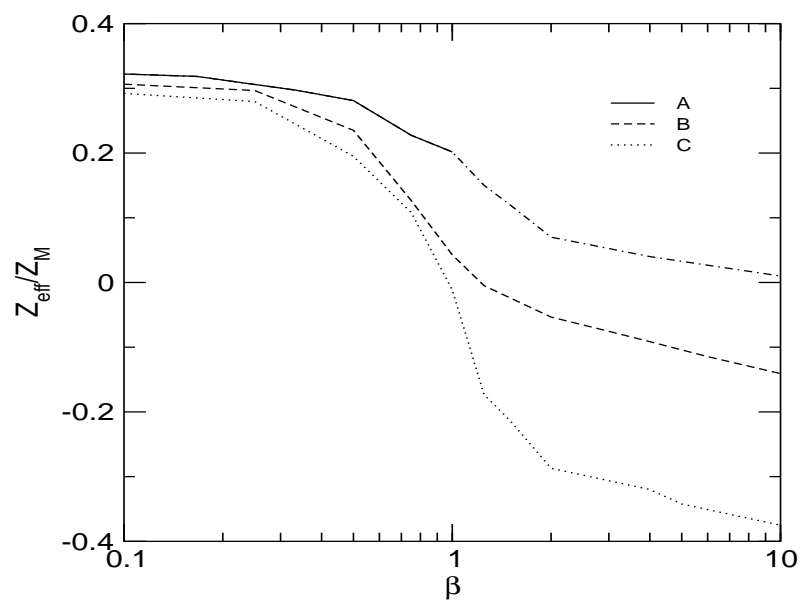

FIG. 5: Effective macroion charge for the series A, B, and $\mathrm{C}$ vs added salt concentration $\beta=Z_{c} \rho_{c} /\left(Z_{M} \rho_{M}\right)$ as obtained from $\mathrm{MC}$ solution of the WS cell model. The data for system A at $\beta>1$ (dotted-dashed curve) are obtained in the region, where the definition of the effective charge based on the inflection point criterion is no longer justified.

values for the stable solutions and the higher ones for the aggregating systems.

The macroion distribution in the bulk solution can be easiest illustrated by simulation snapshots shown in Fig. 6 The snapshot a) corresponds to a low added salt content $\beta=0.1$ and reveals a homogeneous spatial distribution of the macroions. In the snapshot b) corresponding to $\beta=1$ one can see macroion clusters. All the macroions are in aggregated state. Finally, snapshot c) demonstrates that at high salt $\beta=5$ most macroions are free and the solution is back to the stable state. The cluster re-dissolution is in agreement with theoretical predictions [26] and recent simulations for polyelectrolyte-induced a aggregation of colloids and globular proteins [24, 25]. We should note that even at high $\beta$ values occasional particle pairs are formed. To ensure the equilibrium thermodynamic state of the system we performed additional simulations with initial compact configuration of the macroions. The macroions were allowed to move after the equilibration of the ionic subsystem. Then, indeed we observed stable aggregates at $\beta \approx 1$ and re-dissolution of the macroions at the higher salt dose. The parameters of the aggregates were drifting slowly towards their values from the simulation with random initial configuration with an increase of simulation length. The problem with matching the two sets of data for the re-dissolution and the cluster-cluster aggregation is caused by the wide energy barriers originating from the large net charge of the clusters [37]. Although the cluster charge is not growing proportionally to the number of macroions due to the partial counterion release, the cluster charge yet reached values of $2 Z_{M}^{e f f}$ in our systems $\mathrm{B}$ and $\mathrm{C}$ at $\beta>1$.

Figure 17 presents partial macroion-macroion radial distribution functions (rdf) for the three systems at different salt concentrations. In Fig. $7 \mathrm{~h}$, one can see the results for system A, which show gradual decay of the macroion ordering on increasing salt. The peak of the rdf appears initially at $r=\rho^{-1 / 3}$ and has a value of 1.15 , then moves towards shorter separations, while decreasing at the same time and almost disappears at $(\beta>1)$. The trend is readily understood from decreasing the effective macroion charge and growing the screening ability of the electrolyte at the higher salt contents. For the remaining two series $\mathrm{B}$ and $\mathrm{C}$, the trend is very different. Already at $\beta=0.5$, there is a peak near the macroion contact distance (or, more exactly, at $r=\sigma_{M}+\sigma_{c}$ ), which then grows high upon salt addition and reaches value of 60 at $\beta=1$ for the series $\mathrm{B}$, and 150 for the series $\mathrm{C}$. At $\beta>1$ it considerably drops in height but still remains quite high even at $\beta=10$. A secondary peak at $r=2 \sigma_{M}$ corresponding to the second particle layer in a cluster is seen for some of the curves in series B and $\mathrm{C}$ at $\beta>1$. We would like to note the reappearance of the remote peak at $r \sigma_{M}=5$ for the series $\mathrm{C}$ at $\beta=10$. We made also another interesting observation for the system $\mathrm{C}$. At the salt concentrations $\beta \geq 1$, the main peak of the macroion-macroion $\operatorname{rdf}$ shifts towards $r=\sigma_{M}$ and is seen now at $r=1.02 \sigma_{M}$ instead of $r=\sigma_{M}+\sigma_{c}=1.1$. This effect might be related to strengthening the electrostatic depletion contribution to the macroion interaction [9].

We plotted some macroion-macroion partial structure factors for different salt concentrations in Figure 8 The structure factors for the series A again shows decay of the structure on increasing $\beta$. For the two remaining series, the structure factors have initially a peak at $q \sigma_{M}=2$ that corresponds to the inverse mean distance between the macroions and a low value in the long-wave limit. On increasing salt, this peak disappears and two new peaks develop instead at $q \sigma_{M} \rightarrow 0$ and $q \sigma_{M} \approx 7$. The first peak reflects the average macroionic cluster size that grows with the salt addition until $\beta \approx 1$ for series $B$ and $\mathrm{C}$. After that, when even more salt is added, the value of the long-wave peak 


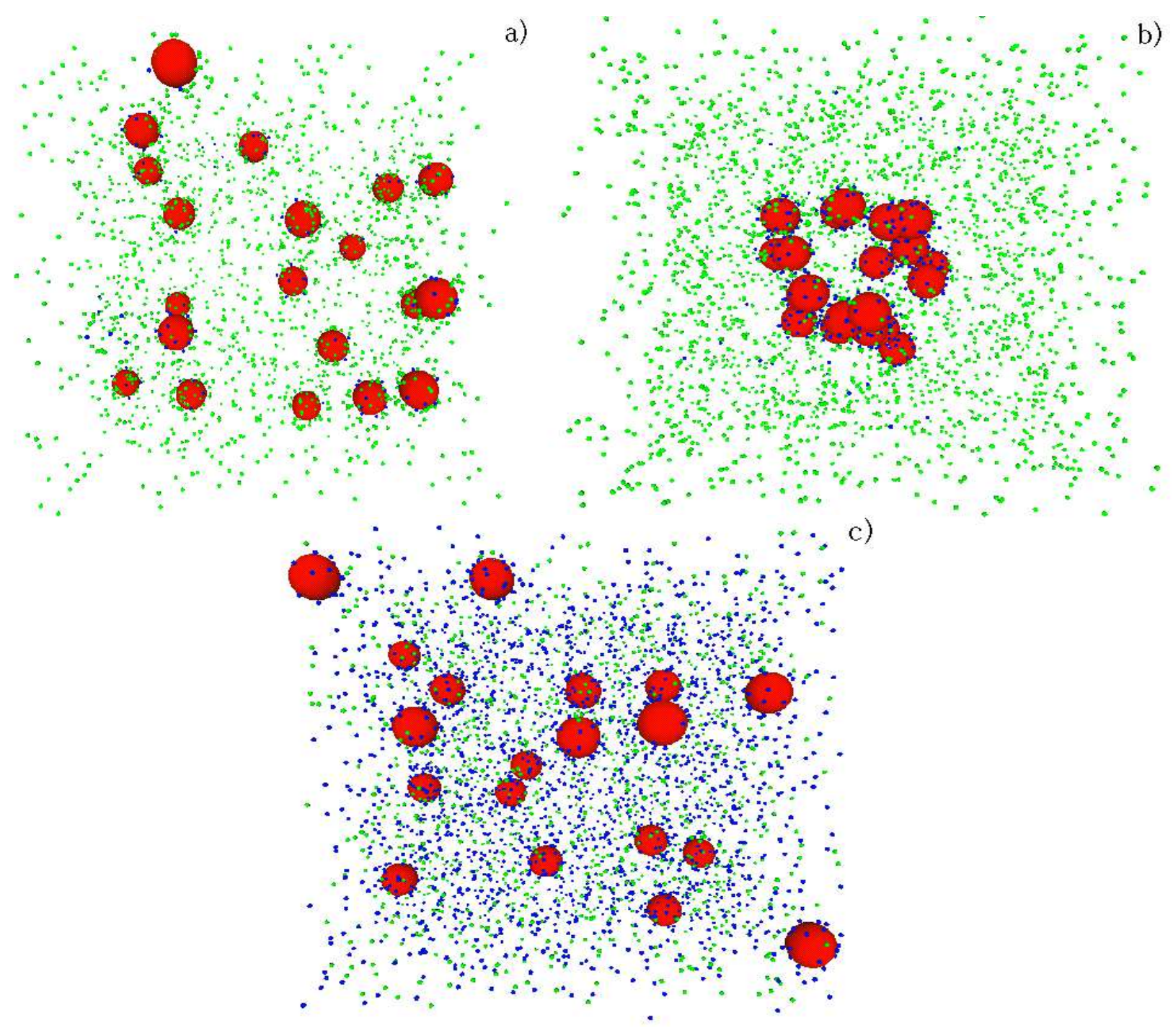

FIG. 6: Snapshots of the primary simulation box for the series $\mathrm{C}$ with 20 macroions taken at the end of simulation at a) $\beta=0.1$, b) $\beta=1$, and c) $\beta=5$. The big spheres are representing the macroions, the small ones representing the counterions and added salt ions. The coions are omitted in c) for clarity. In the snapshot b) corresponding to $\beta=1$, one can see macroion cluster.

decreases, which indicates the re-dissolution of the clusters. The broad secondary peak at $q \sigma_{M} \approx 7$ corresponds to the macroion-macroion density build-up close to contact distance.

The whole set of values of the long-wave structure factor value $S(0)$ for all three series is shown in Fig. 9 The curve for system A shows gradual increase between $\beta=0$ and 6 while the curves for systems $\mathrm{B}$ and $\mathrm{C}$ have well expressed maxima around $\beta=1$. For the system $\mathrm{B}$, the peak is slightly shifted towards higher salt concentration due to the competition between the adsorption energy and the cation entropy, which leads to incomplete macroion charge compensation at $\beta=1$. For the series $\mathrm{C}$, the $S(0)$ value returns to the values lower than 1 , as it was at $\beta \ll 1$. The overall trend for the system $\mathrm{C}$ corresponds to its destabilization at $\beta \approx 1$ a consequent re-stabilization at high salt that occurs due to recovery of the repulsion between the macroions (cf. the effective charge plot in Fig. (5) [15, 26].

\section{DISCUSSION}

As we have seen from the above results, the salt conditions are crucial for the stability of asymmetric electrolytes. On the path along the salt concentration axis, two important parameters of macroion interactions are affected: the Debye screening length and the effective charge of the macroions. The effect of the former is rather smooth and 
can be detected through a simple damping of the interactions upon salt addition. The sensitivity of the asymmetric electrolyte to the added salt depends also on the relative amounts of the "native" and "added" ions. We note that overall properties of the studied systems mark the charge compensation point $\beta=1$ either by an extremum (the osmotic coefficient) or an inflection (potential energy, effective charge), which can be understood as a crossover from the counterion dominated $(\beta<1)$ to the salt dominated screening $\beta>1$.

Another interaction parameter, the effective charge, influences mostly the macroion related properties such as the macroion distribution and the stability of the macroion subsystem. In the counterion dominated regime, the effective charge is changing almost linearly with the salt concentration. This makes the system extremely sensitive to the addition of salt. In contrast, at the higher electrolyte strength the effective charge is increasing logarithmically with the amount of added multivalent salt. We note a close similarity between the observed charge behaviour and the macroion electrophoretic mobility vs added polyelectrolyte dose curves reported in Refs. 3, 4]. Fig. 10 demonstrates the direct relation between the effective charge and the stability of the electrolyte solution. In the plot one can see the relative effective charge curve together with the characteristic of stability $S_{M M}(0)^{-1}$ for the series C. It is important to note the approximate symmetry of the curves in Fig. 10 relative to the isoelectric point, which allows one to formulate the result in a symmetric manner. While one can say that on the right-hand side of the diagram the macroions are overcharged by the excess multivalent counterions, on its left-hand side there is excess of macroions themselves and they are overcharging the multivalent ions. The latter gets a richer physical meaning when we imagine polyelectrolyte molecules instead of the multivalent ions. The effect of the internal counterion structure was discussed recently [22, 24]. As it was shown previously for the asymmetric electrolytes without added salt [28], the parameters of importance are the charge asymmetry between the oppositely charged ionic species and the ion correlation parameter $\Gamma$. Extending our conclusions, we should expect that the strongly charged systems with similar energetic parameters have common features irrespectively of the internal structure of the polyions, whether they are polyelectrolytes or colloids.

While changing the salt valency leads to a smooth variation of the system's properties such as the internal energy, osmotic coefficient, or effective macroion charge at the salt-overdosed conditions far from the isoelectric point, the interactions and structure change drastically in all the series at $\beta=1$. Our results confirm that the instability appears at a certain threshold counterion valency and becomes sharper at the higher counterion charge (or counterion coupling $\Gamma$ ). Therefore, at the higher $\Gamma$ one can expect on one hand a much stronger macroion aggregation at $\beta=1$ and, on another hand, a much better expressed overcharging and re-dissolution effects. Thus, the three systems of our choice correspond to (i) the weak coupling region and counterion entropy dominated behaviour (series A), (ii) border region demonstrating the coexistence of condensed and free counterions at $\beta=1$ (series B) and (iii) the high coupling (or "low temperature") regime with completely condensed multivalent counterions (series C). The alllter case was analyzed in detail recently [26]. At the same time, the instability in real solutions should be much enhanced due to van der Waals forces, which due to their short-range character similarly play a role only in the vicinity of the isoelectric point, i.e. the whole sequence must be shifted towards lower $\Gamma$ values [25].

Finally, we would like to report on the simple salt effect on the effective charge and the counterion correlation attraction. The salt effects in similar systems were discussed previously [21]. It was confirmed that the added salt screens the repulsions between the macroions and leads to weakening the long-range macroion ordering. In a 60:2 electrolyte, addition of divalent ions caused an increase of the electrostatic attraction. In the 60:3 electrolyte, which is similar to our system B but has no monovalent counterions, no significant effect was noticed. Basing on the above analysis, we can explain the increase of the macroion aggregation in the 60:2 electrolyte by that the added divalent ions in fact lessen the effective macroion charge, which is not compensated completely at $\beta=1$ (similar to system $\mathrm{B}$ of the present work at $\beta=1$ ) [38]. The apparent salt effect in the 60:3 electrolyte was small because the considered salt concentrations placed the system in the center of the instability region, where it is not very sensitive to the salt. The salt concentration range in 21] corresponds to $1<\beta<4$ in the current work (cf. the curve for the system B in Fig. 91) Now, we repeat the comparisons of Ref. 21] between the solutions B and C at $\beta=1$ and corresponding systems with multivalent ions only, namely $60: 3$ and 60:5 electrolytes.

In the 60:3 system, the absolute potential energy per macroion is about $2.5 \%$ smaller than in series $\mathrm{B}$, while the difference is about 3.3\% between the 60:5 electrolyte and the system C. Similar relations hold for the partial electrostatic energies: the macroion-cation and cation-cation energies, respectively, are $3.3 \%$ and $5.5 \%$ higher in the $60: 3$ solution than in the $\mathrm{B}$ series at $\beta=1$. For the $60: 5$ solution and system $\mathrm{C}$, these diffrences are marginal: the macroion-cation energy is $0.3 \%$ greater in the former, while the cation-cation energy is the same within the statistical uncertainty. The osmotic coefficient calculated in the cell model in the 60:5 system is equal to zero, which means that all the multivalent ions are adsorbed and the bulk osmotic coefficient is then just $\Pi /\left(\rho_{M} k_{B} T\right) \approx 0.08$. The simulation for the 60:3 electrolyte showed about 5 times higher cation concentration in the bulk for the corresponding system B, though both concentrations were very small. At the same time, the presence of the monovalent ions leads to a very modest decrease in correlation between the multivalent ions. The cation-cation correlation peak in system B falls by about $10 \%$ with respect to $60: 3$ electrolyte, which corresponds to a slight decrease of the effective correlation parameter 
$\Gamma$. In the system $\mathrm{C}$ compared to the $60: 5$ system, the analogous decrease is just about $5 \%$. The macroion-cation rdfs display marginal sensitivity to the added electrolyte. The peak height for the salt-free case is only $5 \%$ higher in the $60: 3$ system, and $2 \%$ higher in the $60: 5$ system than for the corresponding samples B and C.

The relative effective charge $Z_{M}^{e f f} / Z_{M}$ for the both charge asymmetries increases in magnitude with the salt addition. With trivalent ions, it rises from $0.015 Z_{M}$ in $60: 3$ system to $0.03 Z_{M}$ in system B. For pentavalent ions, it changes from 0 in 60:5 electrolyte to $-0.01 Z_{M}$ in system $\mathrm{C}$. Note that the presence of the anion layer around the adsorbed cations does not cancel the effect (see Fig. 3). A significant role here is played by the monovalent counterions, which also accumulate close to the macroion surface. The macroion structure displays considerable sensitivity to the presence of monovalent ions. In the 60:3 system, the first peak of the macroion-macroion rdf increases from 60 to 200 as compared to the system B ( $\mathrm{Fig} 7 \mathrm{~b})$. The structure factor value $S_{M M}(0)$ rises from 2.1 to 13 (Fig $8 \mathrm{~b}$ ). For the case of pentavalent ions, the rdf peak rises from 150 to 230 (Fig 7 ), while the initial structure factor peak from 6 to 13 (Fig 8 ). One can see from the Fig [7bc and Fig [ $8 \mathrm{bc}$ that the aggregation is much stronger expressed in both 60:3 and 60:5 electrolytes than in the corresponding systems B and C. This drastic change can be attributed to (i) the decrease of the effective correlation parameter $\Gamma$ for the multivalent ions in presence of monovalent ones and (ii) the change in the macroion effective charge, which has slightly higher magnitude for the systems with monovalent ions present. The accumulation of these small but finite macroion charges opposes the cluster-cluster aggregation and prevents formation of very large clusters.

\section{CONCLUSION}

We performed a numerical study of a highly asymmetric electrolyte solution containing strongly charged macroions and different amounts of salt. The addition of a simple monovalent salt gradually lowers the effective macroion charge and reduces the solution stability. Addition of small amounts of multivalent salt produces similar effect. The dose of multivalent salt that exceeds the macroion isoelectric concentration causes macroion charge inversion and electrostatically driven macroion aggregation. At the isoelectric point, we observe formation of large macroion clusters. When the inverted macroion charge becomes large enough, the clusters re-dissolve. Enlarging the counterion valency strenghens the instability at the isoelectric point but stabilises the solution at the overdosed conditions. The obtained stability diagram closely resembles polyelectrolyte-induced colloidal aggregation, while the aggregation mechanism is related solely to electrostatic correlation forces rather than the van der Waals attractions.

We are grateful to Per Linse, Boris Shklovskii, Toan Nguyen, Michal Borkovec, Christian Holm, and Peter Schurtenberger for stimulating discussions. This work was financially supported by the Swiss National Science Foundation.

[1] Gregory, J.J. J. Colloid Interface Sci. 1973, 42, 448.

[2] Miclavic, S. J.; Chan, D. Y. C.; White, L. R.; Healy, T. W. Langmuir 1994, 98, 902.

[3] Walter, H. W.; Grant, S. B.Colloids Surf. A 1996, 119, 229.

[4] Bouyer, F.; Robben, A.; Yu, W. L.; Borkovec, M. Langmuir 2001, 17, 5225.

[5] Guldbrand, L.; Jönsson, Bo; Wennerström, H.; Linse, P. J. Chem. Phys. 1984, 80, 2221.

[6] Gronbech-Jensen, N.; Beardmore, K. M.; Pincus, P. Physica A 1998, 261, 74.

[7] Rouzina, I.; Bloomfield, V. A. J. Phys. Chem. 1996, 100, 9977.

[8] Hribar, B.; Vlachy, V. Biophys. J. 2000, 78, 694.

[9] Allahyarov, E.; D’Amico, I.; Löwen, H. Phys. Rev. Lett. 1998, 81, 1334.

[10] Kjellander, R. Chem. Phys. Lett. 1984, 112, 49.

[11] Wu, J.; Bratko, D.; Blanch, H. W.; Prausnitz, J. M. J. Chem. Phys. 1999, 111, 7084.

[12] Linse, P.; Lobaskin, V. Phys. Rev. Lett. 1999, 83, 4208.

[13] Shklovskii, B. Phys. Rev. Lett. 1999, 82, 3268.

[14] Netz, R. R.; Orland, H. Europhys. Lett. 1999, 45, 726.

[15] Nguyen, T. T.; Rouzina, I.; Shklovskii, B.I. J. Chem. Phys. 2000, 112, 2562.

[16] Messina, R.; Holm, C.; Kremer, K. Phys. Rev. Lett. 2000, 85, 87; Phys. Rev. E 2001, 64, 021405.

[17] Grosberg, A. Yu.; Nguyen, T. T.; Shklovskii, B. I. Rev. Mod. Phys. 2002, 74, 329.

[18] Levin, Y. Physica A 1999, 265, 432; Rep. Prog. Phys. 2002, 65, 1577.

[19] Vlachy, V. Annu. Rev. Phys. Chem. 1999, 50, 145.

[20] Hansen, J.-P.; Löwen, H. Annu. Rev. Phys. Chem. 2000, 51, 209.

[21] Linse, P.; Lobaskin, V. J. Chem. Phys. 2000, 112, 3917.

[22] Reščič, J.; Linse, P. J. Phys. Chem. B 2000, 104, 7852.

[23] Skepö, M.; Linse, P. Macromolecules 2003, 36, 508.

[24] Jonsson, M.; Linse, P. J. Chem. Phys. 2001, 115, 10975. 
[25] Carlsson F.; Malmsten M.; Linse P. J. Am. Chem. Soc. 2003, 125, 3140.

[26] Nguyen T. T.; Shklovskii B. I. J. Chem. Phys. 2001, 115, 7298.

[27] Harnau, L.; Hansen, J.-P. J. Chem. Phys. 2002, 116, 9051.

[28] Linse P., Phil. Trans. Roy. Soc. A 2001, 359, 853; J. Chem. Phys. 2000, 113, 4359.

[29] Lobaskin V.; Linse P. J. Chem. Phys., 1999, 111, 4300.

[30] Lobaskin, V.; Linse, P. in Simulation and visualization on the grid of the Lecture notes in computational science and engineering series; Springer: Berlin, 2000; v. 13, p. 165.

[31] Manning, G. J. Chem. Phys. 1969, 51, 924.

[32] Oosawa, F. Biopolymers 1968, 6, 1633.

[33] Belloni, L. Colloids and Surfaces A 1998, 140, 227.

[34] Bocquet, L.; Trizac, E.; Aubouy M. J Chem. Phys. 2002, 117, 8138.

[35] Deserno, M.; Holm, C.; May, S. Macromolecules 2000, 33, 199.

[36] Tanaka, M.; Grosberg, A. Yu. J. Chem. Phys., 2001, 115, 567.

[37] Nguyen, T. T.; Shklovskii, B.I., Phys. Rev. E 2002, 65, 031409.

[38] Lobaskin, V.; Lyubartsev, A.; Linse, P. Phys. Rev. E 2001, 63, 020401. 

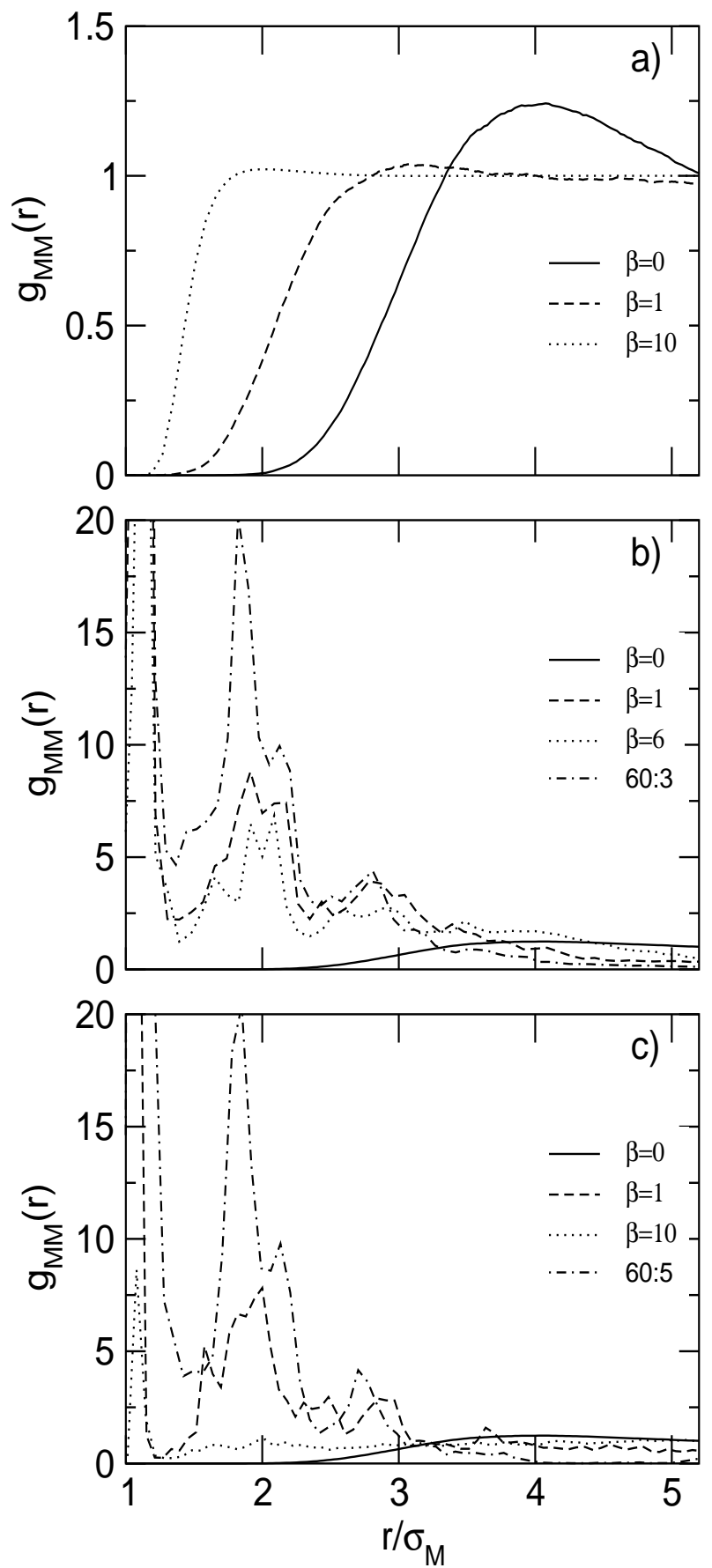

FIG. 7: Macroion-macroion partial radial distribution functions for series A, B, and C, respectively, at the indicated added salt concentrations given in units of $\beta=Z_{c} \rho_{c} /\left(Z_{M} \rho_{M}\right)$ from MC simulation with 20 macroions. The curves obtained for asymmetric electrolytes 60:3 and 60:5 without monovalent ions are given for comparison (see discussion in section [V]. The stripped initial maxima have values of 60 and 150 for the series B and C, and 200 and 230 for 60:3 and 60:5 systems, respectively. 

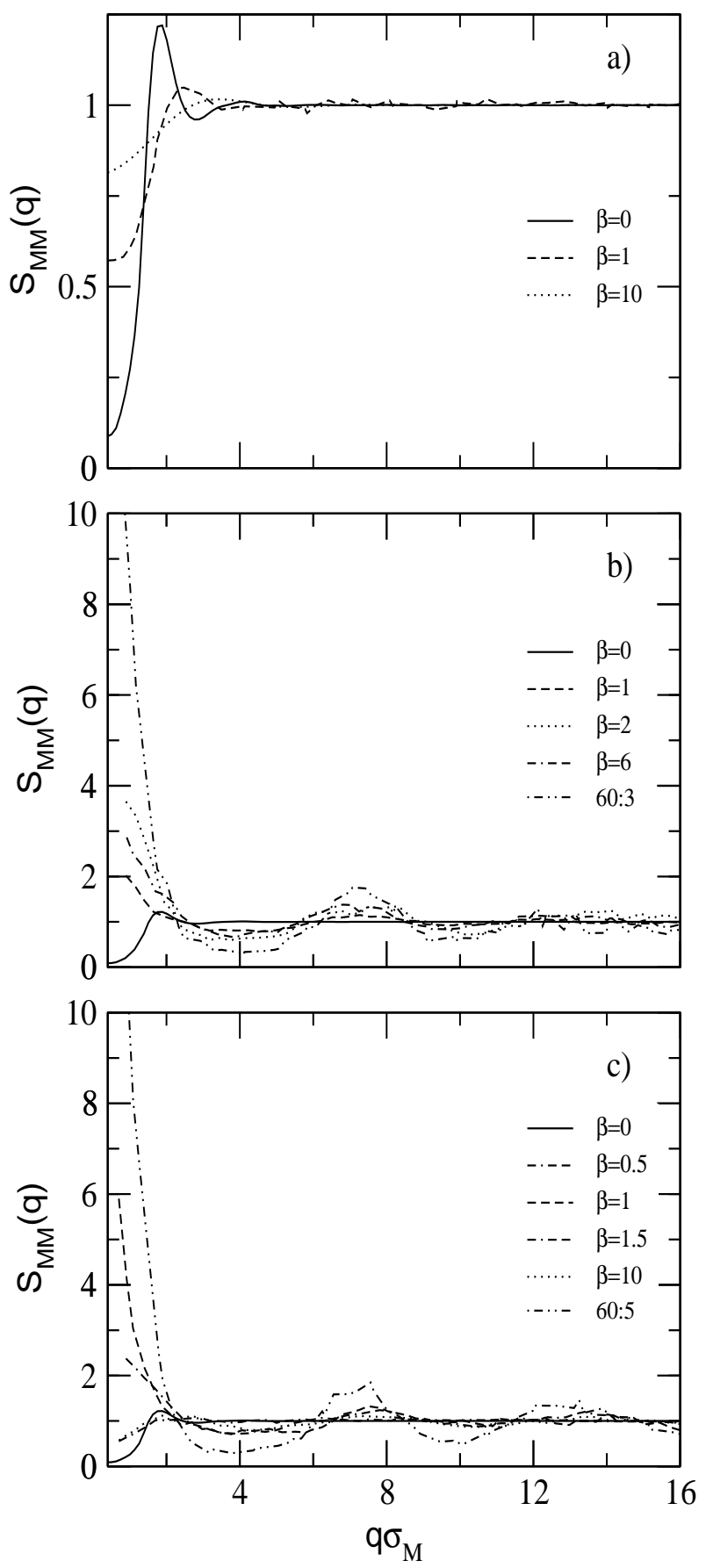

FIG. 8: Macroion-macroion partial structure factors for series A, B, and C, respectively, at the indicated added salt concentrations given in units of $\beta=Z_{c} \rho_{c} /\left(Z_{M} \rho_{M}\right)$ from MC simulation with 20 macroions. The curves obtained for asymmetric electrolytes 60:3 and 60:5 without monovalent ions are given for comparison (see discussion in section [IV. 


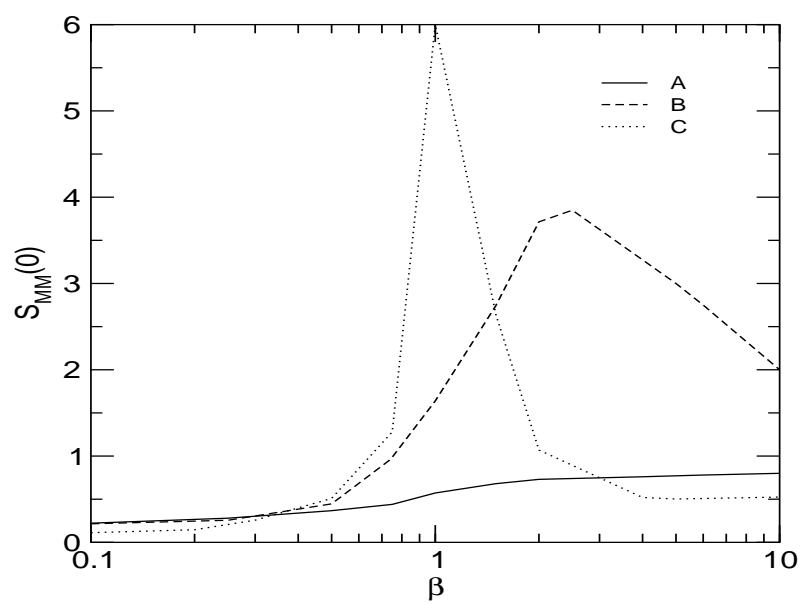

FIG. 9: Long-wave value of the macroion-macroion partial structure factor $\left(S_{M M}(0)\right)$ for the series A, B, and C from MC simulation with 20 macroions.

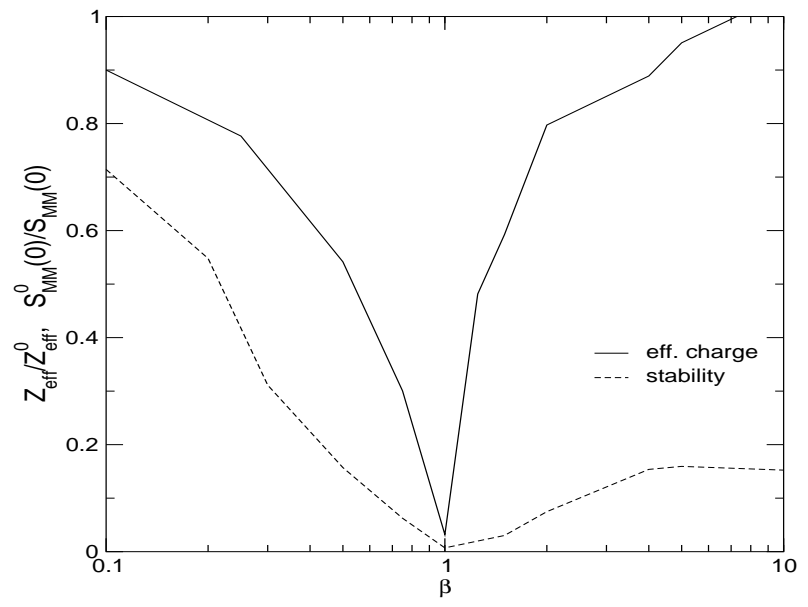

FIG. 10: Absolute value of effective macroion charge and the stability ratio $\left(S_{M M}(0)^{-1}\right)$ for the series $\mathrm{C}$ normalized by their values at $\beta=0$. 